\title{
Cyclic movement execution and its influence on motor programmess
}

\author{
Marcin Dornowski ${ }^{1, A-F}$, Andriy Gorkovenko ${ }^{2, A-D}$, Tomasz Tomiak, ${ }^{1, A-D}$, Viktor S. Mishchenko, ${ }^{1, B-D}$, \\ Dominika Wilczyńska ${ }^{1, B-D}$, Henryk Olszewski ${ }^{3, A-D}$, Piotr Sawicki ${ }^{1, B-D}$, Arkadii N. Talnov ${ }^{2, B-D}$ \\ ${ }^{1}$ Gdansk University of Physical Education and Sport, Gdansk, Poland \\ 2 Department of Movement Physiology, Bogomoletz Institute of Physiology, National Academy of Sciences of Ukraine, \\ Kiev, Ukraine \\ ${ }^{3}$ Institute of Psychology, University of Gdańsk, Poland \\ A - Research concept and design, B - Collection and/or assembly of data, C - Data analysis and interpretation, \\ $D$ - Writing the article, E-Critical revision of the article, F- Final approval of article
}

Dornowski M, Gorkovenko A, Tomiak T, Mishchenko VS, Wilczyńska D, Olszewski H, Sawicki P, Talnov AN. Cyclic movement execution and its influence on motor programmess. Ann Agric Environ Med. 2019; 26(2): 361-368. doi: 10.26444/aaem/94881

\begin{abstract}
Objectives. The aim of this study was to discover the relationship between the performance of different mechanical movements of rowers, and define its effect on the motor programs of the cyclic movement in athletes living in rural and urban areas.

Materials and method. Twenty-two male rowers participated in the experiment using a rowing ergometer (Concept2, USA). The experiment consisted of 3 tests examining the maximal power of the pull-ups (MPbpu). The movement mechanogram was registered with a specialized complex Noraxon's 3D MyoMotion (Noraxon Inc., USA). The software of the complex allowed calculation of the values of the joint angles from the accelerometer data. The Origin Lab 8.5 program was used for the mathematical and statistical processing of the signals from the mechanograms.

Results. It was found that all experiment participants had a stepped controlled increase in the power of single bar pull-ups leading to a corresponding proportional increase in the frequency of rowing - test 1 and, conversely, a stepped controlled increase in the rowing frequency accompanied by a proportional increase in the power of the bar pull-ups - test 2 . The involuntary dependence of the power and the rate was due to the peculiarities of the central cyclic movement programming, according to which the forces and durations of the active and passive bar pull-ups phases were interconnected and regulated together. The voluntary power-rate dependence control led to the breakdown of these links in the motor program of cyclic movements and to the separate control of these parameters.

Conclusions. Motor programs in cyclic movement may be created in the same pattern in tope level sport and recreation, as well in different environmental conditions - gym halls (movement simulators), professional and recreational water sport tracks.
\end{abstract}

Key words

locomotion, mechanics, water sport, environment, rural and urban areas

\section{INTRODUCTION}

Athletic rowing is a complex, specific form of human locomotion that is thought to be one of the most automatic of voluntary actions. Rowing involves the majority of a person's muscle groups (basic muscles of the arms, legs and trunk), as a repetitive sequence of relatively stereotyped movements that combine voluntary and involuntary features. Similar to walking, running and swimming, rowing is mainly controlled by the central motor program (CMP) of cyclic motor activity, which is formed and fixed during training. The question about how much a skilful rower can voluntarily direct the different parameters of rowing is still being debated.

The speed of a rowed boat depends on the power of the bar pull-ups and the pace of rowing. Power is defined as the work performed per unit of time: $\mathrm{W}=\mathrm{dA} / \mathrm{dT}$, where $\mathrm{W}$ = power, $\mathrm{A}=$ work, and $\mathrm{T}=$ time. Hence, the power can be increased either by increasing the work at a constant time of its execution, or by reducing the time it takes to perform

Address for correspondence: Marcin Dornowski, Department of Sport, Gdansk University of Physical Education and Sport, ul. Kazimierza Górskiego 1, 80-335 Gdansk, Poland

e-mail: mdornowski@awf.gda.pl

Received: 20.06.2018; accepted: 05.09.2018; first published: 21.09.2018 constant work, or by simultaneously changing the work and the time in different combinations. The rowing period is divided into two phases: active phase (drive), during which the power is generated, and the passive phase (recovery), during which the oars return to the starting position [1]. With a controlled increase in the rowing frequency, the period is shortened by decreasing the duration of both phases [2]. Reducing the time of the active phase should increase power if the performed work value is maintained. Indeed, real rowing and simulations $[2,3,4,5,6,7]$ have shown that in the frequency range of 20-40 bar pull-ups per minute (st $/ \mathrm{min}$ ), increasing the rowing frequency resulted in a proportional increase in the power and strength of the bar pull-ups.

In the above cases, subjects controlled the frequency of rowing, and the increasing power was a consequence of increased frequency. It was shown [8] that the dependence of power on frequency was reversible in a reciprocal manner: a controlled increase in power was accompanied by a proportional increase in frequency. This result was not trivial, since the power of the bar pull-ups depended on the duration of the active phase, but did not depend on the duration of the passive phase. At the same time, $80 \%$ of the change in the rate of rowing was due to a change in the duration of the passive phase. A priori, it is easy to 
imagine that the increase in the movement frequency could be voluntary in the task of controlled power; however, the revealed features of the CMP of cyclic movements excluded this possibility. The muscle strength and the duration of the active and passive bar pull-ups phases in the CMP of cyclic movements were strictly connected. In the voluntary mode, performing rowing cycles independently, subjects could separately control the duration of the active and passive bar pull-ups phases. As soon as the cycles became merged and the subjects switched to repetitive cyclic motion, control over the active and passive phases was lost. The CMP of cyclic motion controlled the entire movement period, and the parameters of the active phase determined the parameters of the passive phase. Thus, under the condition of cyclic motion, the duration of the passive phase could not be random or voluntary; it depended entirely on the duration of the active phase, which ensured a proportional frequency dependence on the developed power. Despite the identical nature of the frequency-power dependence obtained with a controlled increase in the pace or with a controlled increase in the power, the identity of the CMP of cyclic movements formed in these cases is not obvious. In particular, it is not known whether the ratio of frequency to power is single-valued in the various ways to achieve such a dependence. It is also not known whether a voluntary change in the frequencypower dependence arises when cyclic movements are formed involuntarily by the CMP.

\section{OBJECTIVE}

The aim of this study was to discover the relationship between the performance of different mechanical movements of rowers, and define its effect on the motor programs of cyclic movement in athletes living in rural and urban areas.

\section{MATERIALS AND METHOD}

The research was carried out in the laboratory of the University of Physical Education and Sport in Gdansk, Poland, with the participation of 22 rowers living in rural and urban areas in the Pomeranian district. Mean age $-21.88 \pm 2.57$ years, body weight $-.82 \pm 12.90 \mathrm{~kg}$, body height $-90.59 \pm 7.79 \mathrm{~cm}$ (hereafter $\mathrm{M} \pm \mathrm{SD}$ ). The experimental procedures were carried out in accordance with the Helsinki Declaration of 1964 and its subsequent amendments. Approval for carrying out the experiment was approved by the Local Ethical Commission. All participants in the study were previously informed about the content and procedures of the experiments and agreed to participate.

Experiment tests. The experiment consisted of a series of tests performed on a rowing simulator (Concept2, USA). During performance on the simulator, the subjects could visually track the pace of rowing (number of bar pull-ups per minute) and the power developed within each bar pull-ups on a special monitor. The maximal power of the bar pull-ups (MPbpu) was determined before the beginning of the main experiments. The subjects performed 7 maximally powerful bar pull-ups for that purpose, and checked the power level on the simulator monitor in the process; the rate of rowing was not specified. The average MPbpu from the findings was used to normalize the bar pull-ups values of a given subject in subsequent tests.

The experiment consisted of three tests. The first test, controlled increase in bar pull-ups power, included five stages of $40 \mathrm{~s}$ duration. In each stage, the subjects increased the power of the bar pull-ups in a stepwise manner. They were informed about a target level of the bar pull-ups power at the beginning of every stage. The participants reached $30 \%$, $40 \%, 50 \%, 60 \%$ and $70 \%$ of their averaged MPbpu without specification of the rowing rate.

In the second test, controlled increase in rowing rate, the subjects increased the rowing rate in five stages of $40 \mathrm{~s}$ duration. The subjects were informed about the upcoming rate targets, the values of which were equal to the levels registered in the corresponding stages of the first test. The bar pull-ups power was not specified in this case.

Test 3 was a voluntary change in the power-rate dependence of the values shown in tests 1 and 2 . This test was divided into two parts: test $3 \mathrm{a}$ and test $3 \mathrm{~b}$, which were each split into three 1 min stages. In test $3 \mathrm{a}$, the subjects had to control the power defined by the experimenter in the first (control) stage (c). They had to reduce the power of each bar pull-ups by $10 \%$ in the second stage (d), maintaining the rate shown in stage (c). The subjects had to increase the bar pull-ups power by $10 \%$ in the third stage (i), maintaining the rate shown in stage (c).

In test $3 b$, the subjects had to control the rate specified by the experimenter in the first (control) stage (c). They had to reduce the rate by $10 \%$ in the second stage (d), retaining the bar pull-ups power shown in stage (c).

In the third stage (i), te subjects had to increase the frequency by $10 \%$ relative to the control stage, retaining the power shown in stage (c).

Bar pull-ups power in test $3 \mathrm{a}$ and the frequency in test $3 \mathrm{~b}$ were set based on the results of test 1 with a power control of $50 \% \mathrm{MPbpu}$. In all tests, the external load regulator corresponded to the average level of the latter (the regulatory damper of the Concept 2 was in position 5); the values of the developed power and the rate of rowing were fixed by a special camera. It was assumed that the test was performed correctly if the mean differences between the stage $\mathrm{c}$ (control rowing) and stages $\mathrm{d}$ (decreased value by $10 \%$ ) and i (increased value by $10 \%$ ) were significant for changeable parameters and nonsignificant for those that were unchangeable.

Registration of the mechanogram. The movement mechanogram was registered with a specialized Noraxon 3D MyoMotion complex (Noraxon Inc., USA). The software of the complex allowed calculation of the values of the joint angles from the accelerometer data.

The system recorded the values of 24 joint angles. Six of them described the subject's torso position: angles of the trunk flexion in the sagittal and frontal planes in the lumbar and thoracic areas, as well as the angles of the trunk rotation around the vertical axis in the same sections. The position of the arm in space was described by five values: the angles of flexion/extension in the elbow and shoulder joints, the total angle in the shoulder joint, the angle of rotation of the shoulder segment of the arm along the longitudinal axis and the angle of the shoulder abduction. The position of the leg was described by the angles of flexion/extension of the hip joint, abduction/adduction of the thigh, rotation of the thigh along the longitudinal axis and flexion/extension of the knee 
joint. The angles of the arms and legs were recorded separately for the right and left sides of the body. The beginning of the knee extension was considered the beginning of the bar pull-ups. Maximum knee flexion was regarded as the end of it. The active phase of the bar pull-ups was defined as the time between the start of the knee extension and the end of the elbow flexion. The passive phase went from the end of elbow joint flexion to the maximum of knee flexion. The work performed during one bar pull-up was calculated as the product of the bar pull-ups power and the duration of its active phase. The amplitude of motion was estimated from the knee extension (degrees). For each subject, this value was close to a number in the range of the developed power of 25-70\% MPbpu. The consistency of the movement amplitude made it possible to consider the work performed during the bar pull-ups as the equivalent of the total muscular force.

Mathematical and statistical processing of results. The Origin Lab 8.5 program was used for the mathematical and statistical processing of the signals from the mechanograms. For statistical analysis, the mechanogram values were calculated for each individual bar pull-up. To assess the linear relationships between different indicators, linear regression was used. One-way ANOVA and Bonferroni's post hoc test (Origin Lab 8.5) were used to determine the average values and the reliability of their differences. In addition, the results were statistically compared by using the ANOVA and ANCOVA (detailed description provided in the corresponding parts of the Results). Analysis was carried out using SPSS Statistics 17.0.

\section{RESULTS}

All 22 subjects had a stepped controlled increase in the power of single bar pull-ups leading to a corresponding proportional increase in the frequency of rowing - test 1 and, conversely, a stepped controlled increase in the rowing frequency accompanied by a proportional increase in the power of the bar pull-ups - test 2. Figure 1 summarizes the data from 10 subjects whose rowing rate values at the stages of increasing in test 2 were equivalent to the frequencies shown by the subjects during the stepwise increase in the bar pull-ups power in test 1 . The dependence of power on rate in test 2 was similar to that in test 1 (Fig. 1a); correlation coefficient $-0.755 ; \mathrm{p}<0.001$ for test 1 and $0.728, \mathrm{p}<0.001$ for test 2 . In addition, test 2 kept retained the main dependencies found in test 1 . The power and rate were proportional to the duration of the active bar pull-ups phase (Fig. $1 \mathrm{~b}$ and 1c, respectively). The correlation between the power values and the active phase durations was $0.825 ; \mathrm{p}<0.001$ for test 1 and $0.827, \mathrm{p}<0.001$ for test 2 . The correlation between rate and the active phase duration was $0.933, \mathrm{p}<0.001$ for test 1 and $0.914, \mathrm{p}<0.001$ for test 2 . The durations of the active and passive phases of the bar pull-ups were proportional (Fig. 1d), with correlation coefficients of 0.907 ; $\mathrm{p}<0.001$ for test 1 and $0.840, \mathrm{p}<0.001$ for test 2 .

Despite the similar nature of the dependencies obtained in both tests, they did not coincide with each other; this fact concerns sets of values on the coordinate plane and regression lines. To assess the differences between the two linear regression coefficients, one-factor ANCOVA was performed between different pairs of indices that characterized one bar pull-ups; the factor was the type of test (two levels, T1 and $\mathrm{T} 2$ ), and the covariance was the rowing rate value (Tab. 1).

Table 1. ANCOVA results of bar pull-ups mechanogram

\begin{tabular}{cccc}
\hline \multicolumn{2}{c}{ Values } & \multicolumn{2}{c}{ Coefficient } \\
\hline Independent & Dependent & $F$ & $P$ \\
\hline FrStr & PwN & 4.21 & 0.040 \\
\hline ActStr & PwN & 108.54 & 0.000 \\
\hline ActStr & PasStr & 177.45 & 0.000 \\
\hline ActStr & FrStr & 93.94 & 0.000 \\
\hline
\end{tabular}

FrStr - rowing rate; ActStr - duration of active phase; $\mathrm{PwN}$ - bar pull-ups power; PasStr - duration of passive phase

The results of the ANCOVA confirmed that the regression coefficients between the pairs of indicators shown in Figure 1 significantly depended on the type of test - T1 or T2.

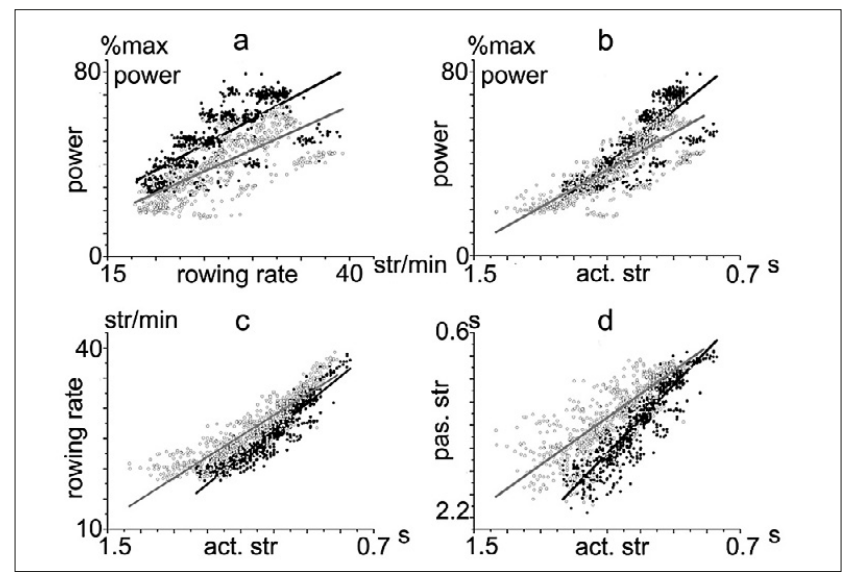

Figure 1. Dependence of bar pull-ups power on rowing rate (a) and on the duration of the active bar pull-ups phase (b). Dependence of rowing rate on duration of active bar pull-ups phase (c). Dependence of duration of passive bar pull-ups phase on the duration of the active phase (d)

The influence of the type of test (T1, T2) and the predetermined rate of rowing on the mechanogram parameters were evaluated by 2-way ANOVA with repeated measurements. The main within-group factors were 1) type of test, which had two levels (T1, T2) and 2) rate of rowing, which had 5 levels. The level of statistical significance was chosen as 0.05 . Bonferroni's post hoc test was chosen for the final estimates of pairwise differences (Tab. 2, Fig. 2).

Table 2. ANOVA results of bar pull-ups mechanogram

\begin{tabular}{lcccccc}
\hline & \multicolumn{2}{c}{ Test } & \multicolumn{2}{c}{ Rate } & \multicolumn{2}{c}{ Test $\times$ Rate } \\
\hline Value & $\mathrm{F}(1,6)$ & $\mathrm{P}$ & $\mathrm{F}(4,24)$ & $\mathrm{p}$ & $\mathrm{F}(4,24)$ & $\mathrm{p}$ \\
\hline FrStr & 0.33 & 0.588 & 472.85 & 0.000 & 0.39 & 0.817 \\
\hline PwN & 31.28 & 0.001 & 528.30 & 0.000 & 7.82 & 0.000 \\
\hline ActSt & 25.21 & 0.002 & 700.70 & 0.000 & 13.63 & 0.000 \\
\hline PasSt & 70.33 & 0.000 & 210.03 & 0.000 & 5.03 & 0.004 \\
\hline TStr & 0.73 & 0.426 & 357.14 & 0.000 & 0.49 & 0.744 \\
\hline WN & 29.56 & 0.002 & 240.16 & 0.000 & 2.79 & 0.049 \\
\hline
\end{tabular}

FrStr - rowing rate; ActStr - duration of the active phase; $\mathrm{PwN}$ - bar pull-ups power; PasStr - duration of passive 
phase; TStr - duration of bar pull-ups; WN - work performed during the active phase.

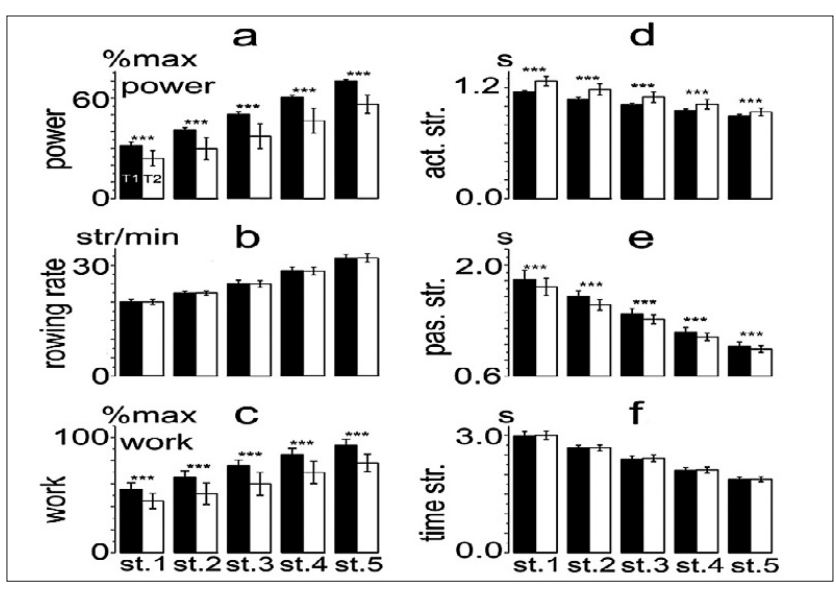

Figure 2. Comparison of average values of bar pull-ups e power (a), rowing rate (b), work of one bar pull-ups (c), duration of active bar pull-ups phase (d), duration of passive bar pull-ups phase (e), total duration of bar pull-ups (rowing period) (f) calculated at each stage of controlled increase of bar pull-ups power (black bars, test 1 ), and controlled increase in rate (white bars, test 2 )

Table 2 shows the ANOVA results of the rowing rate, bar pull-ups power, durations of the active and passive phases and work values for 10 subjects, by combining the results of all participants in five stages in each test (test $1 \mathrm{n}=788$; test $2 \mathrm{n}=801)$. Analysis showed that the values of the rowing pace - rowing frequency and bar pull-ups period - did not depend on the type of test (Fig. 2b, 2f). The indicators of the average rowing power depended not only on the type of test and the rate of rowing, but also on the interaction of these factors. These indicators were significantly higher during $\mathrm{T} 1$ at all five rowing speeds than during $\mathrm{T} 2$, while the difference in the average power index between $\mathrm{T} 1$ and $\mathrm{T} 2$ increased slightly with the rowing rate (Fig. 2a). The average work demonstrated the same tendency (Fig. 2b). The average duration of the active bar pull-ups phase also depended on such factors as the type of test, the rowing rate and their interaction. Furthermore, this parameter decreased as the rowing rate increased. At the same time, its indices in $\mathrm{T} 1$ were significantly lower than in T2 at all rates of rowing (Fig. $2 \mathrm{~d}$ ). The average duration of the passive bar pull-ups phase had the opposite tendency (Fig. 2e).

It should be noted that the average duration of the active phase in test 2 was longer than that in test 1 , just as the average duration of the passive phase was shorter in test 2 than that in test 1 , which actually allowed the rowers to maintain the same average frequency in both tests. As can be seen, even such a rough estimate made it possible to reveal reliable differences in the parameters of the cyclic motion CMP in tests 1 and 2.

The rowing rate in test 1 was increased proportionally in the five stages (Fig. 2b, black bars) with a controlled increase in power in each stage (Fig. 2a, black bars). Athletes reproduced the test values in the five stages of test 2 (Fig. $2 b$, white bars). Moreover, the proportionally increased lowering of the bar pull-ups was significantly lower at all stages than the power shown in test 1 (Fig. 2a, white bars). Two factors underlie the power-frequency ratio decrease in test 2 (rate control). The first was a reduction in the work performed per bar pull-ups (Fig. 2c); the second was the extension of the active bar pull-ups phase (Fig. 2d). Thus, it was found that under rate control, the subjects, showing the same rowing rate as in the power control, rowed with less force, increasing the ratio of the active and passive bar pull-ups phases in favour of the former. To fulfil the condition of rate equality, the CMP of cyclic motion shortened the passive bar pull-ups phase in all five stages of the rate change while prolonging the active phase (Fig. 2e). As a result, the total time (rowing period) of the bar pull-ups in test 2 remained the same as in test 1 (Fig. $2 \mathrm{f}$ ). In test 1 , compared to all stages of test 2 , 20 subjects had greater bar pull-ups power at the same rate of rowing. The average differences, revealed by Bonferroni's post hoc test, were $7.02 \pm 0.582 \%$ MPS with $\mathrm{p}<0.001$ at the rate of $20 \mathrm{st} / \mathrm{min}, 10.42 \pm 0.692 \%$ MPS with $\mathrm{p}<0.001$ at the rate of $22.5 \mathrm{st} / \mathrm{min}, 14.06 \pm 0.905 \%$ MPS with $\mathrm{p}<0.001$ at the rate of $25 \mathrm{st} / \mathrm{min}, 14.20 \pm 0.707 \%$ MPS with $\mathrm{p}<0.001$ at the rate of $28 \mathrm{st} / \mathrm{min}$ and $13.74 \pm 0.872 \mathrm{MPS}$ with $\mathrm{p}<0.001$ at the rate of $32 \mathrm{st} / \mathrm{min}$. At the same pace of rowing, two subjects had the same power in both tests. Thus, in the majority of cases, the bar pull-ups power could differ within $14 \%$ of the MPbpu at the same rate, due to various combinations of the active and passive phase durations in the CMP of cyclic motion. These results suggest that power and frequency can be voluntarily controlled within the limits shown previously.

The 22 subjects had to perform two additional tests (test $3 \mathrm{a}$ and test $3 \mathrm{~b}$ ). Figure $3 \mathrm{a}, \mathrm{c}$ demonstrates examples of the correct execution of test $3 \mathrm{a}$ and $3 \mathrm{~b}$, respectively.

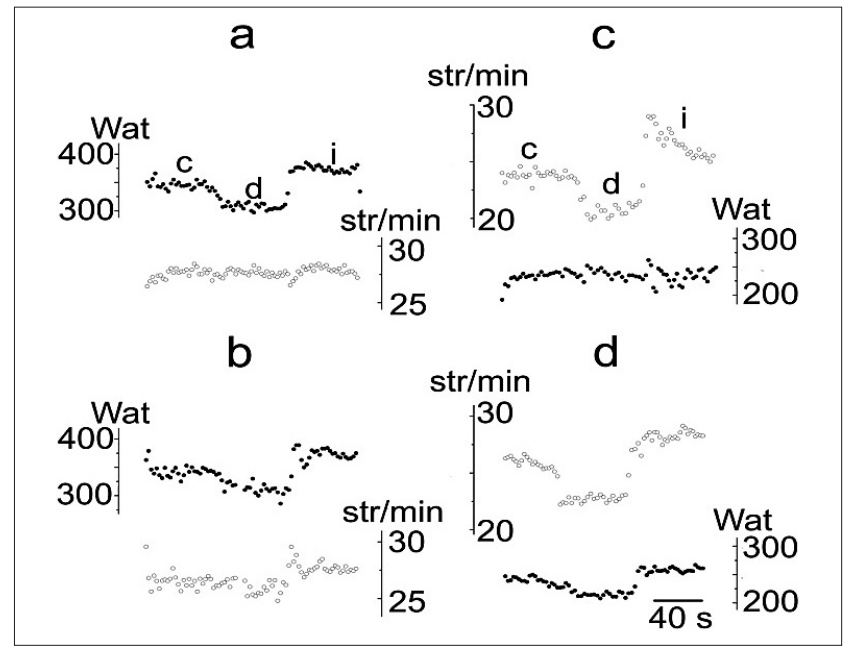

Figure 3. Examples of correct $(a, c)$ and unsuccessful $(b, d)$ performance of tests $3 a$ (change in bar pull-ups power at a constant rate) and $3 \mathrm{~b}$ (rate change at a constant bar pull-ups power), respectively

An incorrect test (Fig. 3b, 3d) was a test in which the average differences between stage $c$ and stages $d$ and i were significant for unchangeable parameters and both significant and nonsignificant for those that were changeable. Only one of the 22 subjects performed the entire test 3 correctly. Four subjects performed test 3 a correctly, and four subjects performed test $3 \mathrm{~b}$ correctly. Most subjects performed only part of the test correctly: either a changeable parameter decreased (d) or increased (i). For this reason, when estimating the number of correctly and incorrectly performed tasks, stages $\mathrm{d}$ and $\mathrm{i}$ of tests $3 \mathrm{a}$ and $3 \mathrm{~b}$ were considered separately. Ten subjects reduced the power without changing the rate, but 12 could not (test 3, a, d). Thirteen subjects increased the power without changing the rate, and nine failed (test 3 , a, i). Ten subjects reduced the rate without changing the power of the bar pullups, but 12 failed (test 3, b, d). Seven subjects increased the 
rate without changing the power, and 15 did not (test $3, \mathrm{~b}, \mathrm{i}$ ). A total of $45.5 \%$ of 88 tests in which one of the parameters (power or frequency) was changed, or the rate remained unchanged, were executed correctly and $54.5 \%$ incorrectly. Approximately $40 \%$ of the tests were performed correctly due to the rupture in the CMP of cyclic movements between the active and passive phases of the bar pull-ups (Fig. 4).

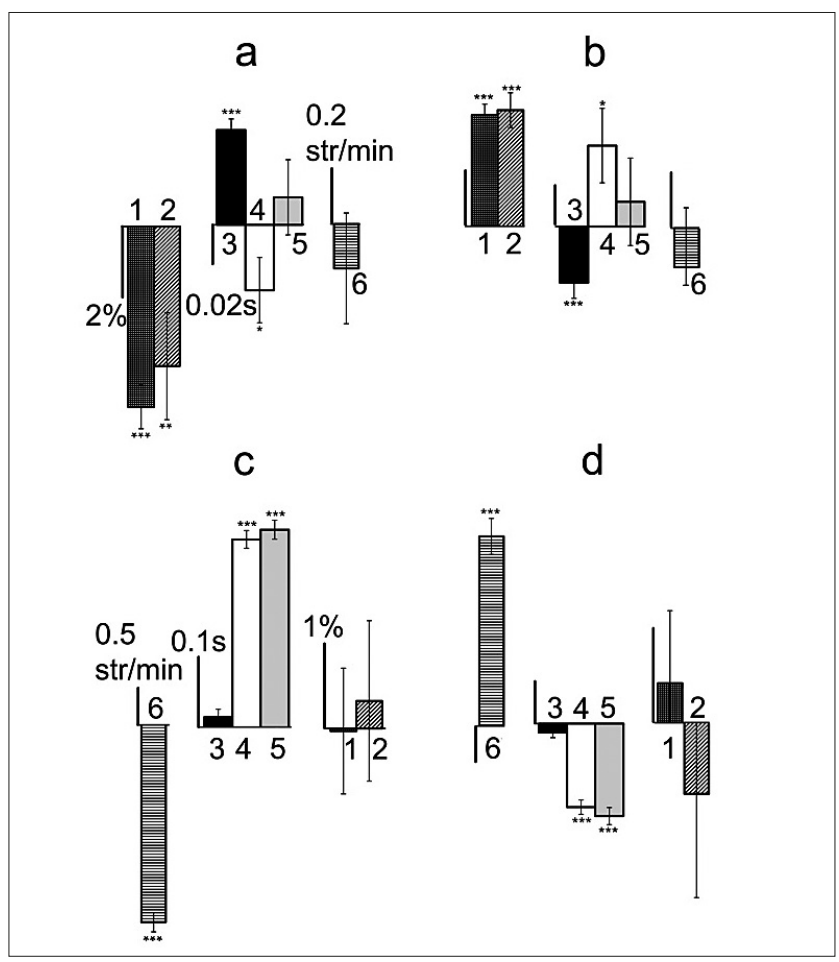

Figure 4. Correct attempts to change voluntarily the power-rate relationship in test 3 by controlling active and passive bar pull-ups phases separately

A change in the power of the bar pull-ups or the rate of rowing in tests 1 and 2 was always accompanied by a unidirectional change in the duration of the active and passive phases of the bar pull-ups. The duration was elongated with decreasing power or frequency, and it was shortened with increasing power or frequency. In the cases shown in Figures 4 a and $4 b$ (test $3 a, 3 d$, and test $3 a, 3 i$, respectively), the durations of the active and passive bar pull-ups phases varied in different directions, i.e., the subjects were able to control the duration of the active and passive phases separately.

Following the instruction to reduce the power without changing the rate, the subjects extended the active phase of the bar pull-ups and reduced the work performed, while in order to prevent a decrease in the rate they shortened the passive phase. This made it possible to keep the rate and the time (period) of the bar pull-ups invariant (Fig. $4 \mathrm{a})$. The same thing happened with a controlled power increase, in an inverse manner: the work was increased and the active bar pull-ups phase was shortened. The passive phase was extended, so the rate did not change (Fig. 4b). In the cases shown in Figure $4 c$ and $4 d$ (test $3 b, 3 d$, and test $3 b$, $3 \mathrm{i}$, respectively), the subjects also managed to control the durations of the active and passive phases separately.

The subjects did not change the duration of the active phase or the amount of work performed by decreasing the rate without changing the power of the bar pull-ups, but extended the duration of the passive phase, which ensured a controlled reduction in the rate (Fig. $4 \mathrm{c}$ ). When increasing the rate without changing the power of the bar pull-ups, the subjects did not change the duration of the active phase or the amount of work performed, but shortened the duration of the passive phase, which ensured a controlled increase in the rate (Fig. $4 d)$. Approximately $40 \%$ of the tests were performed incorrectly because of the subjects' inability to break the relationship between the active and passive bar pull-ups phases in the CMP of cyclic movements (Fig. 5).

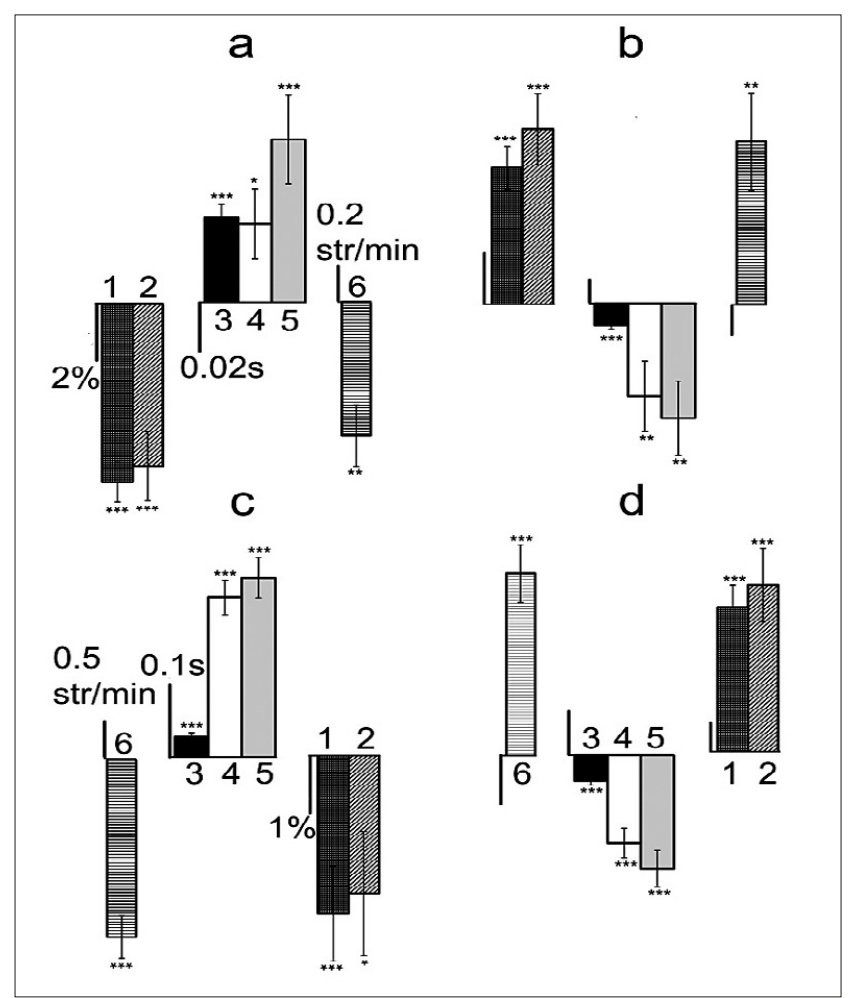

Figure 5. Unsuccessful attempts to change power-frequency dependence voluntarily in test 3 , owing to the inability to control the active and passive bar pull-ups phases separately

In these cases, the durations of the active and passive phases o0f the bar pull-ups varied unidirectionally, as in tests 1 and 2, which resulted in a decreased rate when the bar pull-ups power decreased (Fig. 5a) and in an increase in rate when the bar pull-ups power increased (Fig. 5b). For the same reason, a rate decrease was accompanied by a decrease in the power of the bar pull-ups (Fig. 5c), and increase in rate caused an increase in power (Fig. $5 \mathrm{~d}$ ). The change in the amount of work, which in this case reflected the force of the the bar pull-ups in tests 1 and 2, in a certain way was connected with changes in the duration of the active and passive bar pull-ups phases. The shortening of the duration was accompanied by a force increase, and elongation by its decrease. In situations with differently directed changes in the durations of the active and passive bar pull-ups phases that occurred during test 3 , the change in the bar pull-ups force was associated with the change in the active bar pull-ups phase duration only. With the extension of the active phase, the amount of work decreased (Fig. 4a), the shortening of the active phase was accompanied by an increase in the amount of work (Fig. $4 \mathrm{~b})$. If the duration of the active phase did not change, the amount of work did not change either (Fig. 4c, 4d). Some subjects attempted to perform separate control of the bar 
pull-ups power and rate, breaking the relationship between the bar pull-ups force and the duration of its active phase in the CMP of cyclic motion. In this way, only $2 \%$ of the tests were performed correctly, all of which occurred during test $3 \mathrm{a}$, stage $\mathrm{d}$. While performing the bar pull-ups powerreducing task without changing the rate, the subjects reduced the power by diminishing the work performed and did not change the duration of the active or passive bar pull-ups phase, thus allowing them to maintain the rate unchanged (Fig. 6a).

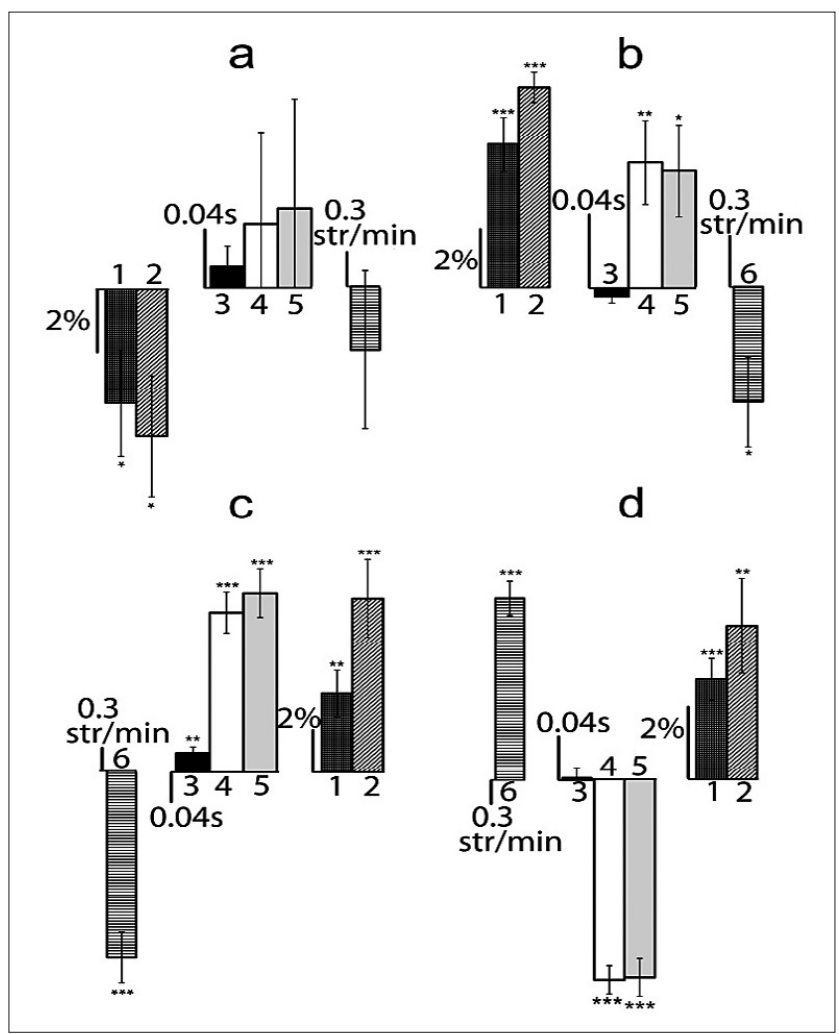

Figure 6. Separate control occurrences of bar pull-ups work and active phase duration while attempting to change the power-frequency ratio voluntarily in test 3

In $3 \%$ of $3 a$ (stage i) tests, the subjects increased the bar pull-ups power by amplifying the work performed without changing the duration of the active bar pull-ups phase, while prolonging the passive phase and reducing the rate (Fig. 6b). In $8 \%$ of $3 \mathrm{~b}$ (stage d) tests, the subjects decreased the rowing rate by increasing the durations of the active and passive bar pull-ups phases, compensating for the bar pull-ups power decrease by elongating the active phase as performance increased.

Finally, the bar pull-ups power increased (Fig. 6c). In $4 \%$ of $3 \mathrm{~b}$ (stage i) tests, the subjects increased the rowing rate by decreasing the duration of the passive bar pull-ups phase. While they kept the active phase duration unchanged, they could not properly control the bar pull-ups force, which led to a power increase in the bar pull-ups (Fig. 6d). In one $3 \mathrm{a}$ test (stage i), the subject, without changing the duration of the active phase, increased power by increasing the amount of work but reduced the duration of the passive phase, which increased the rate. In three more trials, the subjects could not increase the bar pull-ups power while keeping the rate unchanged. In one test, the subject could not reduce the power of the bar pull-ups without changing the rate.

\section{DISCUSSION}

While rowing on the simulator Concept 2 under the instructions of the experimenter, all subjects performed a stepped increase in the bar pull-ups power accompanied by a proportional increase in the rowing rate, or a stepped increase in the rate accompanied by a proportional increase in the power. These data are consistent with previously obtained results $[3,4,5,6,8]$. The power-frequency dependence was similar in both types of trial. However, a rowing rate increase led to decreased durations of the active and passive bar pullups phases [2] and, owing to the shortening of the active phase, to an increase in the power of the bar pull-ups, whereas there was no such evidence in the opposite frequency-power ratio, since the change in the frequency by $80 \%$ depended on a change in the duration of the passive bar pull-ups phase [8]. Bar pull-ups power a priori depends on the duration of the active bar pull-ups phase and force. It was demonstrated that these parameters were connected in the cyclic motion CMP. The shortening of the active phase was accompanied by an increase in the force of the bar pull-ups. The dependence of frequency on power was due to the rigid connection between active and passive phases of the bar pull-ups in the CMP of cyclic movements.

Despite the evident similarity in the above-mentioned dependencies on the power control (test 1) and rate control (test 2), the value of the bar pull-ups power at the same rate was always higher under power control than under rate control. This finding meant that the power-frequency dependence was not invariant, as assumed earlier [8], but relied on the method of its ascertainment. It was found that the differences in the values of the bar pull-ups power under the conditions of tests 1 and 2 were related to the ratio of the active and passive bar pull-ups phase durations. During rate control, the subjects, starting the rowing cycle from the active phase and generated power, the magnitude of which was not explicitly specified. Such circumstance allowed them to minimize energy costs by choosing the maximum possible duration of the active phase for the frequency specified by the experimenter and, accordingly, performing the minimum possible work. During power control, the subjects generated the power set by the experimenter; therefore, the duration of the active phase and work performed were determined. In this case, the frequency was not set explicitly, and the subjects could minimize it by choosing the maximum possible duration of the passive phase for a given duration of the active phase. The results obtained show that the bar pull-ups power at a given rate value was involuntarily minimized within $14 \%$ of the MPS. At the same time, an attempt to change the power/ frequency ratio within $\pm 10 \%$ of the MPS and $\pm 10 \%$ of the rowing rate voluntarily was fully achieved by only one of the 22 subjects; another was able to change the power/frequency ratio within $\pm 10 \%$ of MPS, and a third changed it within $\pm 10 \%$ of the rowing rate. Most of the subjects could change the power/frequency ratio voluntarily in just one part of the test; successful attempts at voluntary control were observed in $45.5 \%$ of the tests, and $54.5 \%$ of such attempts failed. This testifies to the considerable difficulties of voluntary intrusion into the cyclic motion CMP. In $40 \%$ of correctly performed tests, the subjects were able to break the connection between the duration of the active and passive phases. Whereas the decrease in active phase duration in tests 1 and 2 was always accompanied by a decrease in the duration of the passive 
phase, a voluntary reduction or increase in the bar pullups power during maintenance of the constant rate was accompanied by opposite changes in the durations of the bar pull-ups phases. During the rowing rate voluntary decrease or increase at a constant bar pull-ups power, the duration of the passive phase decreased and increased accordingly, but the duration of the active phase did not change, which again confirmed the predominant connection of the bar pull-ups power with the parameters of the active bar pull-ups phase and the rowing rate with the parameters of the passive phase.

Approximately $40 \%$ of the tests were unsuccessful because the subjects could not break the relationship between the parameters of the active and passive bar pull-ups phases in the cyclic movement CMP. In these cases, as shown in tests 1 and 2 , controlled power or rate reduction was accompanied by a combined prolongation of both bar pull-ups phases, and a controlled increase in power or rate led to their shortening. In tests 1 and 2 and in $80 \%$ of the test 3 attempts (successful and unsuccessful), the work change, which was considered as the equivalent of the bar pull-ups force, was associated with changes in the duration of the active bar pull-ups phase. This was highlighted in cases where the parameters of the active and passive phases changed in opposite ways. This result is in good agreement with earlier data $[2,8]$ and confirms the assumption that the involuntary power-rate dependence was the result of the interdependent link between the parameters of the bar pull-ups force and the durations of the active and passive phases in CMP of cyclic movements. In $20 \%$ of test 3 attempts, the subjects broke the connection between the bar pull-ups force and active phase duration. The break of this connection led to the correct execution of the test in only one case.

It is interesting to compare the presented results with studies of other locomotive cyclic movements - walking and swimming. There are synphase and antiphase locomotor movements (SLM and ALM, respectively), in which the former, the left and right sides of the body move in phase and mirror each other. Examples of such movements include rowing, breast bar pull-ups and butterfly bar pull-ups. Examples of ALM are walking, front crawl and back bar pull-ups. One paper that studied walking drew a number of conclusions that coincide with those presented [9] in that walking is an extremely individual form of locomotion, both in kinematics, dynamics, kinetics and EMG of muscle activity, in which either flexor or extensor muscles can dominate. Additionally, the paper noted that inter-individual differences in walking motor control cannot be distinguished by studying standard walking modes, such as step speed and number. In the presented case, no serious differences in the kinematics or kinetics of individual bar pull-ups were noted during simulation of standard 2,000 $\mathrm{m}$ distance. The interindividual differences in motor control were detected only during 'non-standard' tests, when the subjects had to control the rowing rate and power simultaneously.

Based on the walking study and the presented results, it can be assumed that whole-body cyclic movements are regulated by a cascade of central rhythm generators, each of which controls the movements of only a certain segment or extremity. General coordination is carried out on central motor command from the higher structures of central nervous system, in particular from the motor cortex [9]. In ontogenesis, walking is no doubt formed very early, while rowing is trained much later. It is possible that other complex cyclic locomotive movements also involve walking-control mechanisms.

The essential difference between rowing and walking is the fact that a rower moves the left and right sides of the body isodirectionally, whereas a walker moves both sides in phase opposition. This may have certain consequences for the differences in motor control of these two types of locomotion. The CNS may regulate many walking parameters independently of each other [10]. This conclusion was made on the basis of walking on a treadmill in which the movement speed of the right and left legs differed [11]. It was also concluded that the mechanisms of movement control are separate for the left and right legs, however, at the same time, there is evidence that locomotor activity of the left and right lower limbs is formed by the same neural mechanisms [12]. The contradiction with the above cited article can be eliminated by assuming that there may be mechanisms for the individual control of each leg, as well as for coordination with each other. The presented results indirectly confirm this supposition. It is necessary for SLM, such as rowing, to have some "parallelization" and synchronization of mechanisms, controlling the right and left limbs separately. In this case, the role of common mechanisms increases. The heightened role of such common mechanisms perhaps explains the problems encountered while performing the 'non-standard' task of the collaborative control of the rowing rate and power, when CNS is forced to control the duration of the active and passive bar pull-ups phases separately. As mentioned before, only one subject accomplished this task correctly.

Comparison of the presented results with the swimming motor control study is slightly more complicated. Due to the methodological difficulties in recording the mechanograms and EMG of the corresponding muscles, there are not many studies in this field. In addition, most studies are devoted to the front crawl style of swimming [13], which refers to the ALM type resembling walking. Therefore, the motor control might be similar between front crawl swimming and walking [13]. Earlier studies showed that front crawl swimming EMG is less differentiated than that of walking [14].

In connection with the above, it is especially interesting to compare the presented results with those obtained in a study of an SLM, an example of which is breast bar pull-ups swimming [15]. In this study, it was proposed that the swimmers reproduce certain patterns of synchronization between the movements of the arms and legs. The corresponding phase relationship was controlled with feedback sound signals. It was found that the most convenient swimming mode was when the arms and legs moved in the opposite phase with a 180 degree phase difference between them, which was close to the natural coordination of the upper and lower extremities without feedback signals. Performing swimming at other values of the phase difference, especially at 90 degrees, was challenging for the subjects. The study concluded that this complexity arose as a result of the possible features of the neural control, as well as of the differences in the anthropometric and dynamic characteristics of the subjects' body segments and limbs. A similar difficulty during 'nonstandard' tasks was also observed in the current experiments.

There were no significant differences between results achieved between the participants from rural and urban areas, which was also of interest to the authors of this study. Rural participants achieved higher results in averaged value of seven maximally powerful bar pull-ups performed from 
the beginning of the experiment. This phenomenen may be similar to a widely known paper describing the level of motor abilities performance of subjects from rural and urban areas [16].

The study of individual peculiarities of kinematics and kinetics when performing 'non-standard' tests can be useful in coaching work for the development of individual training plans. Gawda et al. have demonstrated that change in sEMG amplitude during isometric exercise could be related to muscles fatigue [17]. The use of various indices [18] and those based on sEMG amplitude, as an objective indicators of the efficacy of training process in athletes and motor programming phenomenon in humans requires further research. In the opinion of the authors of the presented study, the microgenetic theory which describe motor programming on the basis of findings in modern neurosciences (i.e., from gene expressions, electrical and chemical language of the brain, endomarkers, neuromarkers to behaviour), might be helpful in interpreting the efficacy of the training process and motor programming phenomenon in athletes living in rural and urban areas $[19,20]$.

\section{CONCLUSION}

The involuntary dependence on the power and the rate was due to the peculiarities of the central cyclic movement programming, according to which the forces and the durations of the active and passive bar pull-ups phases were interconnected and regulated together. The voluntary powerrate dependence control led to the breakdown of these links in the motor program of cyclic movements, and to the separate control of these parameters.

Motor programs in cyclic movement may be created in the same pattern in top level sports and recreation, as well in different environmental conditions - gym halls (movement simulators), professional and recreation water sport tracks.

\section{Acknowledgement}

The research was supported by Grant 0024/RSA2/2013/52 from the Ministry of Science and Higher Education program 'Rozwój Sportu Akademickiego', Warsaw, Poland.

\section{REFERENCES}

1. Guével A, Boyas S, Guihard V, Cornu C, Hug F, Nordez A. Thigh muscle activities in elite rowers during on-water rowing. Inter J Sports Med. 2001; 32(02): 109-116.
2. Hofmijster MJ, Van Soest A J, De Koning JJ. Gross efficiency during rowing is not affected by stroke rate. Med Sci Sports Exercise. 2009; 41: 1088-95.

3. Di Prampero PE, Cortili G, Celentano F, Cerretelli P. Physiological aspects of rowing. J Appl Physiol. 1971; 31(6): 853--857.

4. Kleshnev V. Comparison of on-water rowing with its simulation on Concept 2 and RowPerfect machines. Proceedings of XXIII international symposium on biomechanics in sports. 2005; pp. 130-133.

5. Steer RR, McGregor AH, Bull AMJ. A comparison of kinematics and performance measures of two rowing ergometers. J Sport Sci Med. 2006; 5(1): 52 .

6. Hofmijster MJ, Landman EHJ, Smith RM, Van Soest AJK. Effect of bar stroke rate on the distribution of net mechanical power in rowing. J Sports Sci. 2007; 25: 403-11.

7. Cerne T, Kamnik R, Vesnicer B, ESganecGros J, Munih M. Differences between elite, junior and non-rowers in kinematic and kinetic parameters during ergometer rowing. Hum Mov Sci. 2013; 32(4): 691-707.

8. Tomiak T, Gorkovenko AV, Mishchenko VS, Korol A, Bulinski P, Vereschaka IV, Tal'nov AN, Vasilenko DA. Control of the Power of Stroke and Muscle Activities in Cyclic Rowing Movements (a Research using Rowing Simulators). Neurophysiol. 2016; 48(4): 1-15.

9. McCrimmon CM, Wang PT, Heydari P, Nguyen A, Shaw SJ, Gong H, Chui LA, Liu CY, Nenadic Z, Do AH. Electrocorticographic Encoding of Human Gaitinthe Leg Primary Motor Cortex. Cerebralcortex. 2017; $1-11$.

10. Rabin E, Shi P, Werner W. Gait parameter control timing with dynamic manual contact or visual cues. J Neurophysiol. 2016; 115: 2880-92.

11. Choi JT, Bastian AJ. Adaptation reveals independent control networks for human walking. Nature Neurosci. 2017; 10: 1055-62.

12. McNeely ME, Earhart GM. Evidence for limb-independent control of locomotor trajectory. Experimental Brain Res. 2010; 201: 613-8.

13. Martens J, Figueiredo P, Daly D. Electromyography in the four competitive swimming stroke: a systematic review. Journal of electromyography and kinesiology: official journal of the International Society of Electrophysiological Kinesiology. 2015; 25: 273-91.

14. Martens J, Daly D, Deschamps K, Fernandes RJP, Staes F. IntraIndividual Variability of Surface Electromyography in Front Crawl Swimming. PloS one. 2015; 10: e0144998.

15. van Houwelingen J, Roerdink M, Huibers AV, Evers LLW, Beek PJ. Pacing the phasing of leg and arm movements in breaststroke swimming to minimize intra-cyclic velocity fluctuations. PloS one. 2017; 12: e0186160.

16. Szopa J, Mleczko E, Żak S. Podstawy antropomotoryki. Warszawa Kraków, PWN, 2000.

17. Gawda P, Ginszt M, Ginsz A, Pawlak H, Majcher P. Differences in myoelectric manifestations of fatigue during isometric muscle actions. Ann Agric Environ Med. 2018; 25(2): 296-299.

18. Tomczak A, Dąbrowski J, Mikulski T. Psychomotor performance of Polish Air Force cadets after 36 hours of survival training. Ann Agric Environ Med. 2017; 24(3): 387-391.

19. Pąchalska M, MacQueen BD, Cielebąk K. Creative potential of microgenetic theory. Acta Neuropsychol. 2018; 16(2): 125-155.

20. Gotner K, Gotner R, Pedrycz-Wieczorska A, Wilczyńska D, Jastrzębowska G, Dornowski M. Hyperbaric therapy influence on psychomotor abilities effectiveness development - two cases study. Acta Neuropsychol. 2018; 16(2): 213-219. 